\title{
An analysis of the performance of foams used in hurling helmets
}

\author{
K. Norris \& S. Tiernan \\ Department of Mechanical Engineering, Institute of Technology Tallaght, \\ Dublin, Ireland
}

\begin{abstract}
Head injuries can occur in most sporting activities. The seriousness of these injuries can vary greatly depending on the sport. Many sports such as American football, cricket, baseball and hurling try to reduce this risk by designing helmets that are suitable to be worn by the players.

The National Standard Authority of Ireland (NSAI) has recently made great strides in this area with the introduction of the first comprehensive standard adopted for the sport I.S. 355:2006, which sets out new testing procedures that all new helmets must meet. The Gaelic Athletic Association (GAA) has also stated its intention to make the wearing of sports helmets compulsory up to minor level. The hope is that in time all the players will become comfortable with wearing helmets which has been found to be a major reason for the current low wear rates.

This paper covers the testing and modelling of energy absorbing materials to ascertain the optimum thickness, and density of foam within the helmet. Static tests are carried out on a compression test machine to obtain stress/strain properties. The foams are tested on a purpose built drop test rig; the displacement, impact force, velocity and acceleration are measured using LabView and a high speed camera with TEMA analysis software to gain a greater understanding of the impact mechanics of each material.

The foams are modelled, both statically and dynamically, using finite element analysis. The static model uses the ANSYS implicit solver, while the dynamic model uses ANSYS/LS-DYNA to create and validate an appropriate foam material model. The displacement, velocity, and energy results from the finite element model and the test results are analysed and compared so that the optimum density and thickness of foam can be obtained.
\end{abstract}

Keywords: head injuries, impact testing, finite element analysis. 


\section{Introduction}

The game of hurling is one of the fastest field games in the world and is also Europe's oldest field game. It is similar to hockey in that it is played with a small ball and a wooden stick and the ball may be struck along the ground or in the air. With the game played at such high speed and intensity, and players being challenged with wooden sticks, a player can receive multiple head impacts during a single game, it is therefore apparent that the helmet must be designed to be a multi-impact helmet

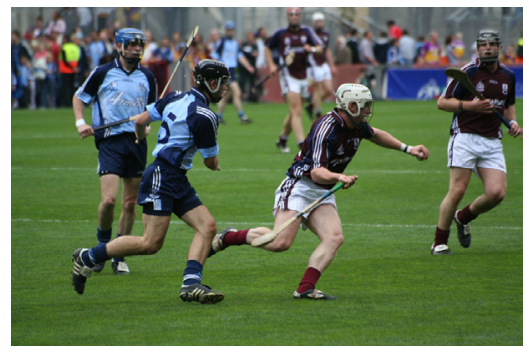

Figure 1: $\quad$ Picture of action from a hurling game.

The National Standards Authority of Ireland (NSAI) has recently introduced a totally new standard, I.S.355:2006, which is intended to improve the effectiveness of hurling helmets. The standard specifies a maximum allowable peak acceleration of $300 \mathrm{~g}$ [1]. To achieve this new standard, helmet manufacturers are being forced to improve their helmet designs. To improve the helmet design a more in depth understanding is required of how the head needs to be protected and the performance of the energy absorbing materials.

Table 1: Reasons for non-wearing of hurling helmets.

\begin{tabular}{|l|l|l|}
\hline Reason & No. of Responses & $\mathbf{\%}$ \\
\hline It is uncomfortable & 26 & 41 \\
\hline It is not necessary & 2 & 3 \\
\hline It feels awkward & 15 & 24 \\
\hline It impairs vision & 27 & 43 \\
\hline It is too expensive & 0 & 0 \\
\hline I play less well with the helmet on & 7 & 11 \\
\hline It looks stupid & 0 & 0 \\
\hline Other & 2 & 3 \\
\hline
\end{tabular}

There has been considerable research carried out worldwide on helmet performance but very little has been done for hurling helmets. This research has indicated that helmets are an effective way of preventing head injury. However, 
as of yet, the wearing of helmets is not compulsory at senior levels of hurling. Flynn et al [2] found that the main reasons for not wearing helmets are that the helmets are uncomfortable and impede the players' vision.

\section{Mechanisms of foam}

To achieve the goal of keeping acceleration below those specified in the I.S. 355:2006 it is necessary to gain an understanding of the mechanisms of foam during an impact. The key to having a correctly functioning helmet is to have the correct density and thickness of foam. If the foam is too thin it will bottom out and have minimal effect in lowering accelerations and forces.

Without the helmet shell, the impact energy would be focused on a small area of the foam, so the shell helps to dissipate the energy over the complete surface area. This helps in preventing the foam from bottoming out during the impact.

Figure 2 illustrates a sample stress-strain graph obtained from the compression tests that were carried out. The graph can be broken into 3 distinct parts: the linear region, the stress plateau and the densification area. It is the stress plateau region that determines how much energy a foam can absorb [3].

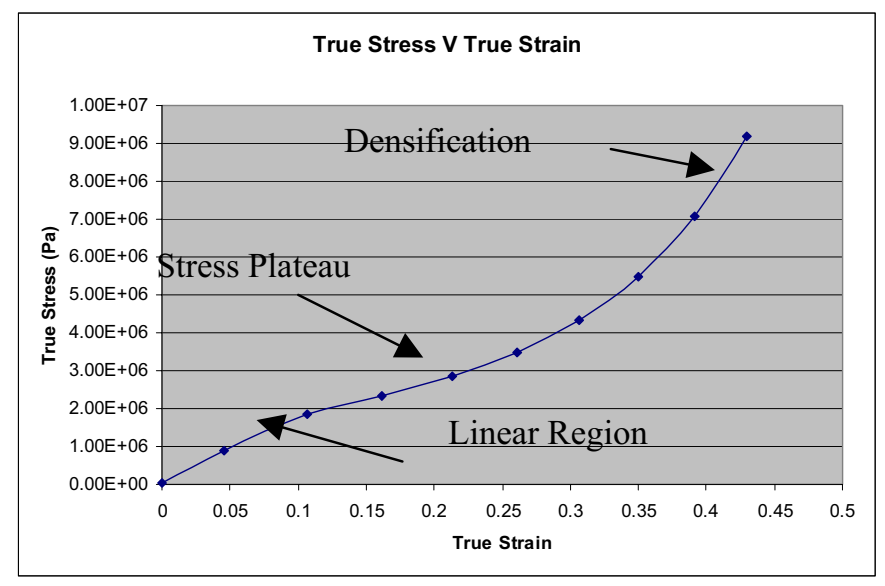

Figure 2: $\quad$ True stress-true strain curve from compression testing.

Figure 2 is the stress-strain graph obtained from the compression testing of a $5 \mathrm{t}-5$ specimen $\left(186 \mathrm{~kg} / \mathrm{m}^{3}\right)$ at a strain rate of $180 \mathrm{~mm} / \mathrm{min}$.

There are two main types of foam, open celled and closed cell foams. The main difference, as seen in Figure 3 and Figure 4, is a membrane covering the cell faces in closed cell foams. In an open cell foam, the fluid is expelled during compression (viscous dissipation) while the membrane in closed-cell foams results in the fluid also being compressed which stores energy that is recovered once the foam is unloaded [4]. 


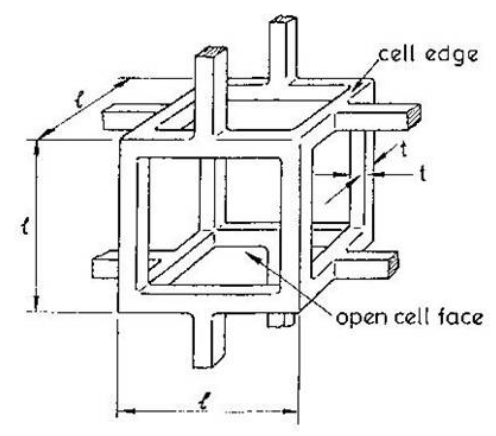

Figure 3: $\quad$ Open cell foam [4].

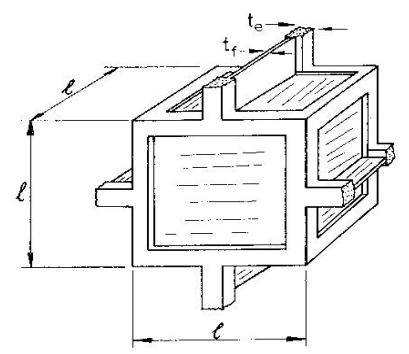

Figure 4: $\quad$ Closed cell foam [4].

\section{Testing}

\subsection{Material data}

Table 2: High density material information.

\begin{tabular}{|c|c|c|}
\hline Specimen & Material & Density $\left(\mathrm{kg} / \mathrm{m}^{3}\right)$ \\
\hline 1 & $3 \mathrm{t}-2.5$ & 398 \\
\hline 2 & $3 \mathrm{t}-3$ & 363 \\
\hline 3 & $3 \mathrm{t}-5$ & 288 \\
\hline 4 & $3 \mathrm{t}-6$ & 246 \\
\hline 5 & $5 \mathrm{t}-2.5$ & 147 \\
\hline 6 & $5 \mathrm{t}-5$ & 186 \\
\hline 7 & $5 \mathrm{t}-7$ & 158 \\
\hline
\end{tabular}

\subsection{Compression testing}

Each material was subjected to two sets of tests: a compression test and a dynamic impact test. Compression tests were carried out on the Instron machine. Each material was compressed to a maximum of $50 \%$ strain as set out in the I.S.355:2006 and the load and displacement were recorded. These results were used to construct true stress- true strain curves for each material, which were then used in creating and validating the material model in the computer simulations.

\subsection{Dynamic testing}

Dynamic impact tests were carried out on a vertical drop impact rig. The impactor falls under gravity and is guided by rails using low friction linear bearings. A $500 \mathrm{~g}$ accelerometer and a force transducer were used to record acceleration and force data. 
A high speed camera was also used to record each drop. Using TEMA analysis software, it was possible to calculate displacement, velocity and acceleration. The high speed camera and National Instruments Labview were used to record the various data from each test. Both systems recorded the acceleration and Labview was used to validate the high speed camera results. By doing this, it was then possible to use the velocity and displacement figures from the high speed camera.

A total of almost 200 impact tests were carried out at a variety of impact energies to understand the impact behaviour of the foam and analyse the influence of density and material thickness on the results. The specimens were also impacted with and without a 'shell piece' to replicate the influence of the helmet shell.

\subsection{Computer simulation}

Computer simulations were carried out using ANSYS. The Compression tests were modeling using ANSYS 11.0 implicit solver where it was possible to compare the simulated stress/strain curve and displacement results with those obtained from testing.

The impact tests were modeled using ANSYS with the LS-DYNA explicit solver. The foam was modeled using the inbuilt closed cell foam model. For this to function correctly, the stress/strain curve from the compression results (see Figure 2) was inputted along with the material properties shown in Table 3.

The impactor, table and shell piece were all modeled as linear materials, with the density, Poisson's ratio and Young's modulus inputted.

Table 3: $\quad$ Material properties inputted to computer model.

\begin{tabular}{|c|c|}
\hline Foam density & $186 \mathrm{~kg} / \mathrm{m}^{3}$ \\
\hline Poisson's Ratio & 0.1 \\
\hline
\end{tabular}

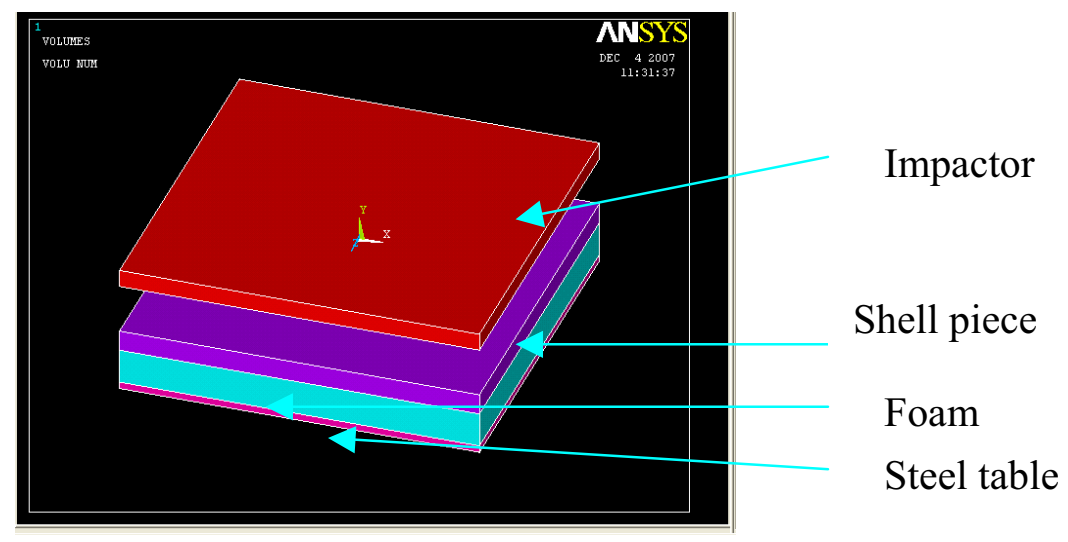

Figure 5: Screenshot of ANSYS model. 


\section{Results}

An integral part of the results analysis was to obtain the transfer function of the impact device. During normal testing, only one accelerometer was used. This was situated at the top of the impact unit. The proposal for this part of testing was to establish whether there were any acceleration loses within the unit itself. For these tests, a $2^{\text {nd }}$ accelerometer was placed on the rig near the impacting head and the readings from the two were compared over a range of impact energies as shown in Figure 6. As seen in Figure 6, the transfer function was found to be 1.

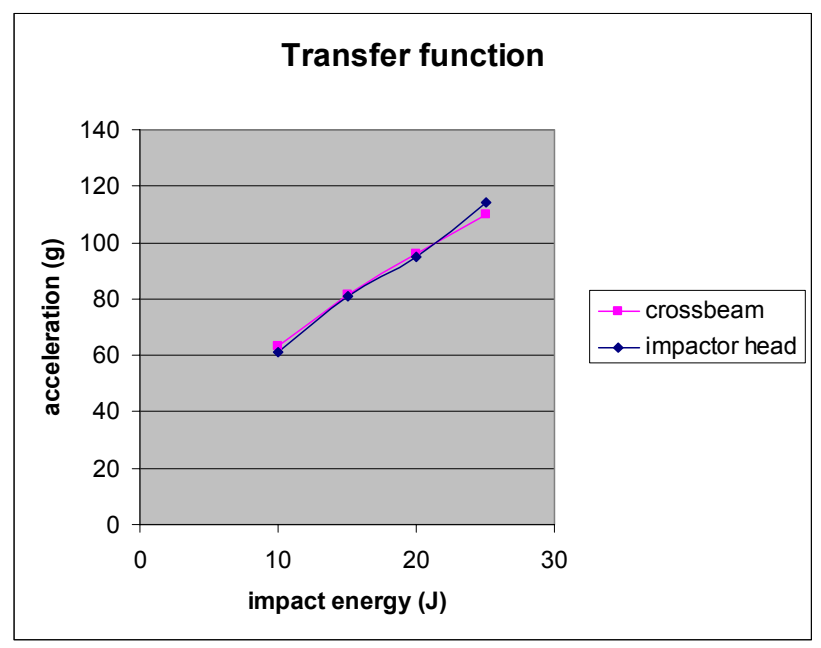

Figure 6: Acceleration $\mathrm{v}$ impact energy to find the transfer function of the crossbeam.

Figure 7 illustrates the effect that the addition of the shell piece has on the force recorded during impact. Examining Figure 7, it can be seen that the shell piece has a significant influence on the force levels. As expected, the shell reduces the force of the impact in all the tests by spreading the force over the entire area of the specimen.

Since the addition of the shell piece, replicates the effect of the helmet shell the following results will concentrate on the 15 Joule tests that were carried out with the shell piece. Figure 8 details the performance of the high density materials over a series of thicknesses.

Upon examining the graph, it can be seen that at the minimum $5 \mathrm{~mm}$ thickness all the foams behave extremely similar to one another. The reasoning behind this is because the foams are compressing so much that they are behaving like solid materials.

However as the thickness increases, the performance differences between the specimens are much easier to observe. The accelerations associated with the $3 \mathrm{t}$ specimens do not show a significant decrease over the range of thicknesses, whilst the $5 \mathrm{t}$ specimens have halved the accelerations recorded at the maximum thickness. 


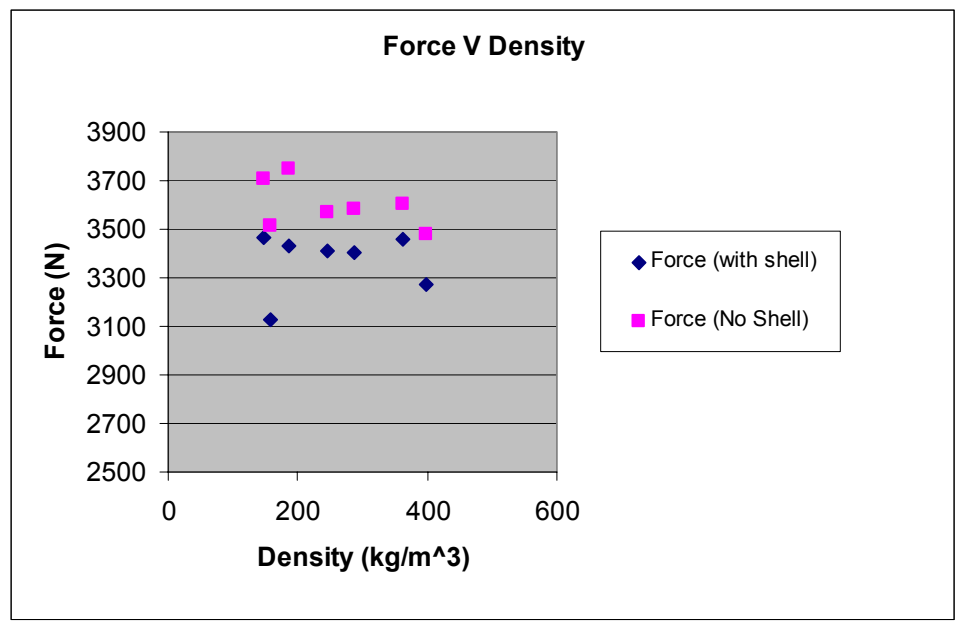

Figure 7: $\quad$ Force v density for $15 \mathrm{~J}$ impact.

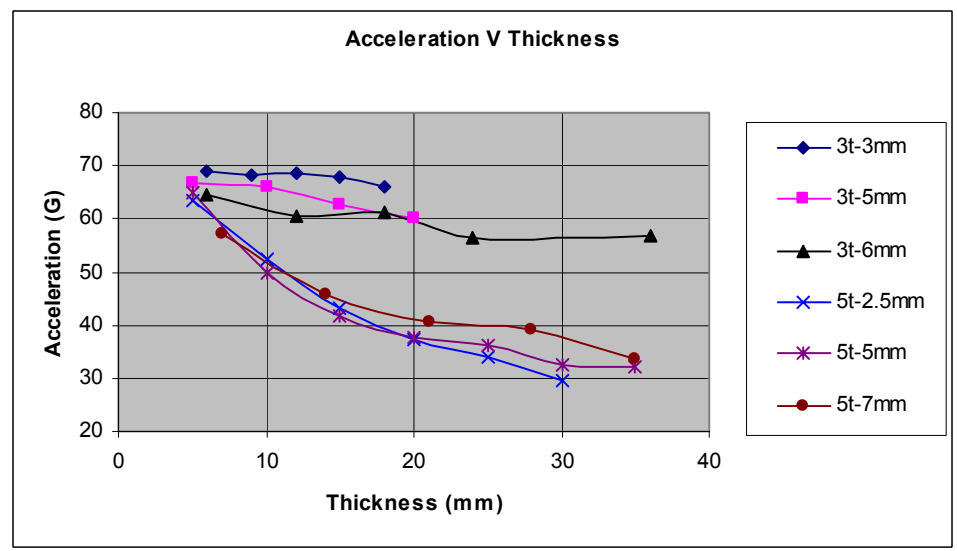

Figure 8: Acceleration $\mathrm{v}$ thickness for high density materials.

Figure 8 gives a great insight into the behaviour of the foams over a range of thicknesses; the maximum thickness of $35 \mathrm{~mm}$ is unrealistic for use in the helmet. It has previously been determined that the maximum allowable thickness of foam in the helmet will be between 5 and $10 \mathrm{~mm}$.

Figure 9 and Figure 10 examine the behaviour of some of the foams in this more suitable region in greater detail.

Figure 9 shows the acceleration results for the specimens impacted at 15 joule impact energy. As the graph illustrates, at a $5 \mathrm{~mm}$ thickness there is not much difference between the performances of the foams. As with Figure 8 however, the performance of the $5 \mathrm{t}-5$ specimen starts to illustrate the performance gains at the $10 \mathrm{~mm}$ thickness. 


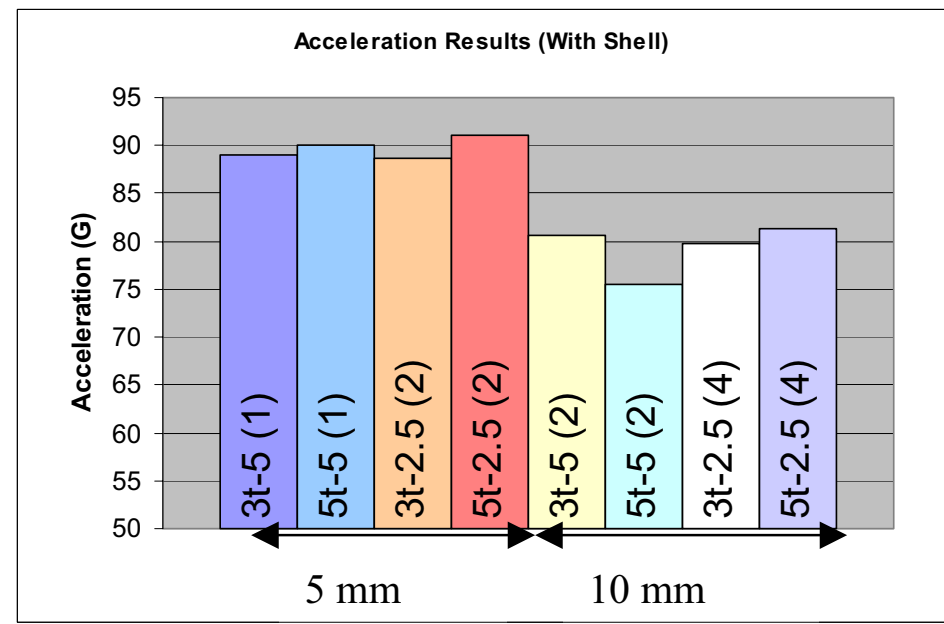

Figure 9: Acceleration results for 15J impact.

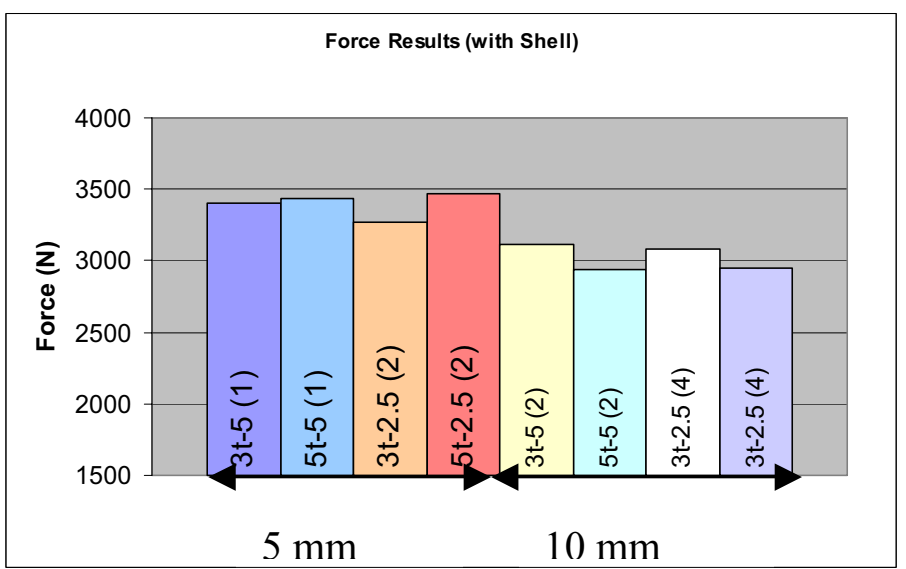

Figure 10: Force results for 15J impact.

Figure 10 illustrates the forces associated with the impact at $5 \mathrm{~mm}$ and $10 \mathrm{~mm}$ thickness. As with the previous graph, there is not much difference between the performances of the foams at this level, however at the $10 \mathrm{~mm}$ thickness the $5 \mathrm{t}$ specimens again show that they have a more significant performance improvement.

Figure 11 and Figure 12 compare the results for the ANSYS simulation with those from the actual tests. Figure 11 compares the energy of both, and on examining these figures they can be seen to have an excellent correlation to one another. The test energy was calculated from the velocity figures obtained from the high speed camera. $\left(E=1 / 2 \mathrm{mv}^{2}\right)$. The initial energy for both is just over 


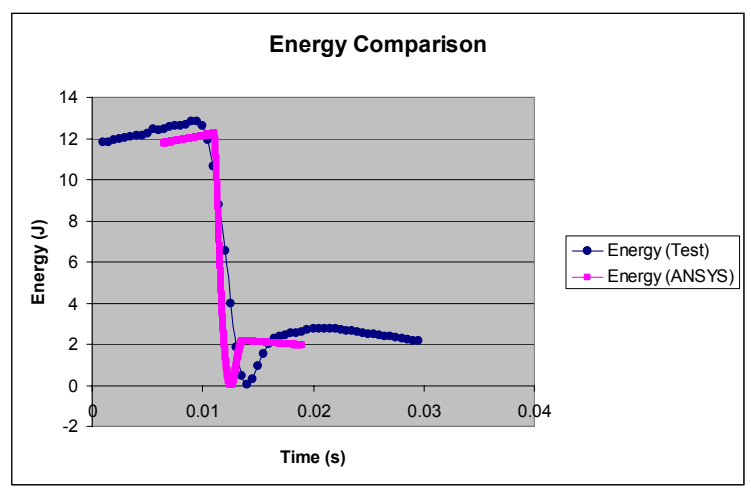

Figure 11: Energy comparison between simulation and test.

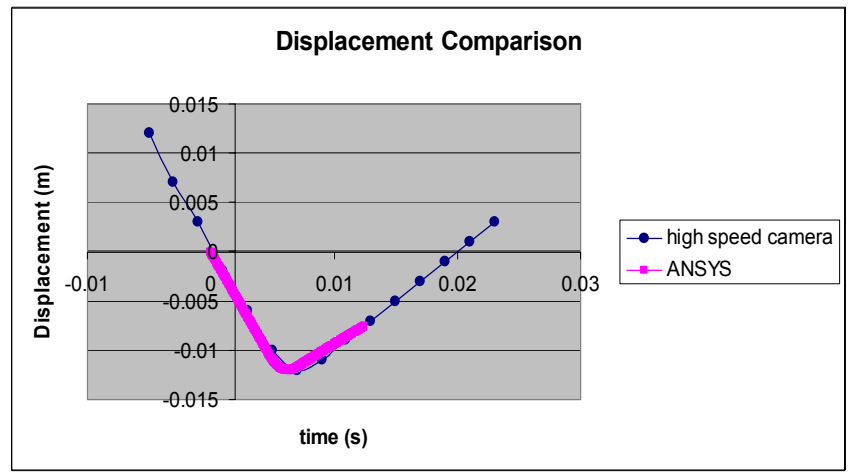

Figure 12: Displacement comparison.

12 Joules and the rebound energy is around 2 Joules. The energy absorbed during the impact is simply calculated by subtracting the rebound energy from the initial impact energy.

The displacement comparison (Figure 12) shows the displacement of the impactor during the impact and this will be used to construct force $\mathrm{V}$ displacement graphs at a later stage in the research. Again there is an excellent correlation between the simulation and the test.

\section{Conclusion}

Figure 8 shows that although there is little difference in performance at the minimum $5 \mathrm{~mm}$ thickness of foam, the $5 \mathrm{t}$ specimens provide the best performance improvement as the thickness increases and this is supported from the results in Figure 9 and Figure 10. From the data compiled, the best performing foams have a density between $150-200 \mathrm{~kg} / \mathrm{m}^{3}$ and this corresponds to the $5 \mathrm{t}$ specimens. 
At the minimum thickness of $5 \mathrm{~mm}$ the foams all behave similarly due to the significant amount of compression that occurs. Therefore, the thickness required for the helmet would be between 7 and $10 \mathrm{~mm}$ of energy absorbing materials. With the computer simulation material model now validated, this will be utilised for the full helmet simulation and then for the development of a new lighter helmet with an increased level of protection.

\section{References}

[1] National Standards Authority of Ireland "Specification for helmets for hurling and camogie players", Published March 2006

[2] Flynn T. H., Fennessy K., et al “Ocular Injury in Hurling”, Br J SportsMed, 39, 2005, 493-496, www.bmjjournals.com

[3] Shuaeib F. M., et al "A new motorcycle helmet liner material: The finite element simulation and design of experiment optimization", Materials and design, 28(1), 2007, 182-195

[4] Lorna J. Gibson \& Michael F. Ashby, Cellular Solids, Structure and Solids $2^{\text {nd }}$ edition, Cambridge University Printing 